\title{
ESTUDIOS
}

\section{Razones de la asimetría del tiempo ${ }^{1}$}

\author{
Reasons for the assimetry of time \\ SEBASTIÁN ÁLVAREZ TOLEDO \\ Universidad de Salamanca
}

Recibido: 27-11-2007 Aprobado definitivamente: 17-01-2008

\begin{abstract}
RESUMEN
En este artículo trata de dos explicaciones de la asimetría del tiempo. En primer lugar, resumo el problema de explicar tal asimetría en términos de procesos termodinámicos, discuto dos intentos de solucionar este problema, y defiendo que, no obstante, no todos los procesos irreversibles han de ser considerados termodinámicos. En segundo lugar, rechazo que la asimetría del tiempo sea simple producto de nuestra condición de agentes intencionales. Finalmente concluyo que la explicación de la asimetría del tiempo que se basa en la multitud y diversidad de los procesos naturales irreversibles sigue siendo una buena explicación.

PALABRAS CLAVE

PROCESOS IRREVERSIBLES, ENTROPÍA, FLECHAS DEL TIEMPO, CAUSALIDAD
\end{abstract}

\begin{abstract}
In the present work I deal with two accounts of the asymmetry of time. Firstly, I summarize the problem of explaining this asymmetry in terms of thermodynamic processes, discuss two approaches proposed to solve this problem, and defend that, nevertheless, not all irreversible processes have to be conceived as thermodynamic ones. Secondly, I reject the notion that the asymmetry of time is a mere product of our condition as intentional agents in the world. Finally, I conclude that the explanation for the asymmetry of time based on the existence of many and diverse irreversible natural processes is still a good explanation.

\section{KEY WORDS}

IRREVERSIBLE PROCESSES, ENTROPY, TIME ARROWS, CAUSALITY
\end{abstract}

1 Este trabajo se ha llevado a cabo en el marco del Proyecto de Investigación DGI HUM2006-04964/FISO del Ministerio de Educación y Ciencia.

(C) Contrastes. Revista Internacional de Filosofía, vol. XIV (2009), pp. 7-22. ISSN: 1136-4076

Licenciatura de Filosofía, Universidad de Málaga, Facultad de Filosofía y Letras

Campus de Teatinos, E-29071 Málaga (España) 
LA PROPIEDAD MÁS CARACTERÍSTICA del tiempo es su asimetría, la inviolabilidad de la orientación antes-después. Mientras que las dimensiones del espacio se nos muestran transitables en los dos sentidos y podemos deshacer en ellas el camino recorrido, ya sea hacia un lado, hacia arriba o hacia al frente, en el tiempo sólo cabe avanzar hacia lo que llamamos el futuro. Podemos configurar en parte lo que está por venir, pero es imposible recuperar o cambiar el pasado. Sin embargo, aunque la asimetría del tiempo resulta evidente en nuestra experiencia, su explicación suscita serios problemas y es objeto de una prolongada polémica filosófica. Es sensato pensar que tal explicación debe encontrarse en el tiempo mismo, ${ }^{2}$ si no es así, en la naturaleza y en lo que ocurre en ella, o tal vez en nosotros, resultando tener un carácter meramente subjetivo. En este artículo me limitaré a analizar algunos aspectos de estas dos últimas posibilidades. Comenzaré considerando la explicación que hace depender la asimetría del tiempo de la existencia de múltiples procesos naturales irreversibles y luego analizaré brevemente la hipótesis que defiende $\mathrm{H}$. Price, según la cual, esta asimetría es tan sólo producto de nuestra condición de agentes, es decir, de nuestra capacidad de transformar intencionalmente nuestro entorno.

\section{LA IRREVERSIBILIDAD EN LA NATURALEZA}

Un modo de explicar el sentido único de la orientación antes-después consiste en hacer dependerla de la existencia de multitud de procesos naturales temporalmente irreversibles, o sea, procesos en los que un sistema pasa por una serie de estados sucesivos sin volver a ninguno de ellos. Y hay en la naturaleza una gran variedad de tales procesos. En el nivel físico, tal vez los más familiares sean los procesos termodinámicos o de incremento de entropía, como, por ejemplo, el paso del calor de los cuerpos más calientes a los más fríos, los fenómenos de mezcla y difusión o las reacciones químicas. Otros procesos físicos irreversibles son los de propagación de ondas. Las ondas emitidas desde un foco se expanden en circunferencias concéntricas y nunca observamos el fenómeno inverso, esto es, la concentración coordinada de las ondas una vez propagadas. Y, a escala cosmológica, la expansión del universo también constituye, al menos en algunos modelos, un camino sin retorno. Por otra parte, es evidente la irreversibilidad en múltiples procesos biológicos, tanto

2 La forma más habitual de concebir la asimetría del tiempo como una característica del tiempo mismo se corresponde con la noción de paso o flujo del tiempo, según la cual, un momento privilegiado, el presente, se desplaza en un único sentido convirtiendo los sucesos futuros en presentes primero y luego en pasados. Sobre la características y problemas conceptuales de esta idea de un tiempo en movimiento se pueden consultar: R. Le Poidevin 1998; D. H. Mellor 1998, capítulos 1-7; y D. Dieks 2006. 
en la evolución y diversificación de las especies, como en el nacimiento y desarrollo de los individuos. Y en animales superiores, la vida mental nos muestra una amplia diversidad de procesos irreversibles. Nuestros conocimientos más ciertos son normalmente sobre el pasado (sobre el futuro sólo nos es posible un conocimiento inferencial) $\mathrm{y}$, en general, nuestras actitudes hacia pasado son muy diferentes de nuestra actitudes hacia el futuro.

Los procesos irreversibles más estudiados en la ciencia son los termodinámicos, y la teoría que se ocupa de ellos, la termodinámica, surgida a mediados del siglo XIX, fue, como se suele decir, la teoría que introdujo la flecha del tiempo en la física. En efecto, la mecánica newtoniana, no distingue entre una y otra orientación del tiempo porque sus leyes fundamentales son invariantes respecto a la inversión temporal, lo que implica que, dada una secuencia de estados de un sistema (por ejemplo, un movimiento) es perfectamente posible, según estas leyes, la secuencia temporalmente invertida, con lo cual el sistema siempre puede volver a su estado inicial. ${ }^{3}$ Y lo mismo cabe decir de las leyes básicas de la mecánica relativista o la mecánica cuántica. Sin embargo, la irreversibilidad de la termodinámica, su asimetría temporal, presenta serios problemas teóricos. Como es sabido, tal asimetría depende en su segunda ley, según la cual, en la evolución espontánea de un sistema aislado, la entropía, esto es, su incapacidad para producir trabajo, nunca decrece y alcanza su valor máximo en los estados de equilibrio. Pero, a pesar de sus éxitos experimentales y tecnológicos, la termodinámica perdió pronto su autonomía teórica y explicativa con el renacimiento de la teoría cinética del calor, según la cual, los fenómenos térmicos se pueden explicar en términos de agitación molecular. ${ }^{4} \mathrm{De}$ este modo la termodinámica quedaba reducida a mecánica o, más precisamente, a mecánica estadística, que es la extensión de la mecánica clásica que se ocupa de sistemas, como los gases, cuya evolución es el resultado de los movimientos aleatorios de multitud de diminutas partículas. Debido a esta reducción, la termodinámica pasó a convertirse en una teoría fenomenológica, es decir, una teoría cuyas leyes y variables (temperatura, presión, volumen) son macrofísicas y es autónoma en este nivel, pero cuya explicación reside, en definitiva, en una realidad microfísica. Por otra parte, esta reducción supuso una ampliación del campo de la ley de entropía, que acabó acogiendo fenómenos irreversibles tan

3 Es decir que para toda solución admitida por las ecuaciones básicas del movimiento, hay otra solución igualmente admisible que es resultado de cambiar el signo, positivo o negativo, de la variable t, lo que se traduce en la inversión de las velocidades y, por tanto, del sentido del movimiento. Para un análisis detallado de las distintas interpretaciones de la invariancia respecto a la inversión temporal, véase S. F. Savitt (ed.) 1995, Introduction.

4 Esta hipótesis fue formulada ya en el siglo XVII, pero cayó luego en desuso por distintas razones, entre ellas, el fracaso de las explicaciones mecanicistas de los fenómenos eléctricos, magnéticos y químicos. 
distintos como la difusión de los gases, las mezclas, los fenómenos de fricción y viscosos, las colisiones inelásticas o las reacciones químicas y nucleares.

Pero la reducción de la termodinámica a mecánica estadística suscitaba interrogantes de difícil respuesta. Dado que, como hemos visto, las leyes fundamentales de la mecánica son invariantes respecto a la inversión temporal, parece que estamos obligados a admitir la posibilidad de inversión temporal en los fenómenos termodinámicos. Es decir, habría que admitir como perfectamente explicables fenómenos tan inauditos como el flujo del calor del cuerpo más frío al más caliente o la separación espontánea de los componentes de una mezcla. Sin embargo ¿cómo es que estos fenómenos no ocurren nunca espontáneamente? ¿cómo es que los fenómenos termodinámicos son irreversibles si los movimientos moleculares que los explican son, como todos los movimientos, reversibles? La solución que propuso el físico austriaco Ludwig Bolztmann a finales del siglo, tan ingeniosa como insatisfactoria, consistía básicamente en lo siguiente. Los estados de los sistemas de que se ocupa la termodinámica son estados macroscópicos caracterizados principalmente por las propiedades de temperatura, presión y volumen, mientras que los estados microscópicos subyacentes se caracterizan por la distribución y las velocidades de las moléculas que componen el sistema. Pero en cualquier sistema son posibles muchísimos más microestados que macroestados, de modo que cada macroestado se puede realizar de múltiples maneras distintas, o sea, mediante múltiples microestados diferentes. Sin embargo, existen grandes diferencias entre macroestados en cuanto al número de microestados que los realizan, y lo que Boltzmann afirmaba era precisamente que el número de microestados que corresponden a situaciones de equilibrio o cercanas a él es abrumadoramente mayor que el de los que implican desequilibrio, de modo que, dado un sistema en un momento determinado y admitiendo que todos sus posibles microestados tienen la misma probabilidad de ocurrir, resulta abrumadoramente más probable que el sistema evolucione hacia un macroestado de mayor o igual equilibrio que hacia uno de menor. De este modo, aunque a nivel molecular el sistema evoluciona de forma aleatoria, sin ninguna orientación definida, fenomenológicamente exhibe una clara tendencia al incremento de entropía (L. Boltzmann 1877). Se trata de una forma de conjugar la simetría temporal propia de la mecánica estadística con la asimetría fenomenológica, macroscópica, de la segunda ley de la termodinámica.

Pero esta solución tenía sus problemas, como señaló Culverwell, porque, al estar basada en una versión estadística de la segunda ley, no podía excluir la posibilidad de descensos espontáneos de entropía y, con ellos, de inversiones de los procesos termodinámicos. Es más, estos fenómenos no sólo serían posibles sino que debían acabar sucediendo en un tiempo suficientemente prolongado, como advertía Zermelo en 1896 retomando un teorema de Poincaré de 1889. Y existía además un problema que afecta a toda versión probabilista de la ley de 
entropía y del que el mismo Bolztmann fue muy consciente. Dada la simetría temporal de las leyes de la mecánica, el razonamiento de Boltzmann no sólo es aplicable en el sentido del futuro, sino también hacia el pasado. Así, dado un sistema en un momento determinado, es tan abrumadoramente probable que evoluciones hacia un macroestado de igual o mayor equilibrio como que haya evolucionado desde una situación de ese tipo. Las mismas razones que avalan el incremento de entropía en el sentido del futuro postulan una mayor entropía en el pasado, que, sin embargo, no es real. Y prescindir de estas consideraciones de simetría en la valoración del argumento de Boltzmann a favor de la irreversibilidad termodinámica, dándolo por válido cuando se aplica en una orientación temporal, pero no en la otra, equivaldría a cometer la «falacia del doble criterio» (Price), a no respetar una elemental «paridad de razonamiento» (Sklar), o a dar por supuesta, en la aplicación del argumento, la asimetría del tiempo que éste pretende demostrar. ${ }^{5}$

Todas estas dificultades de la explicación de la irreversibilidad termodinámica ${ }^{6}$ han llevado a algunos físicos y filósofos a concluir que la imagen del tiempo coherente con la física fundamental es un tiempo simétrico, carente de una orientación privilegiada, de modo que los fenómenos termodinámicos irreversibles de nuestra experiencia habrán de ser considerados fenómenos aislados, locales, debidos a excepcionales fluctuaciones de las condiciones cósmicas generales. El mismo Boltzmann acabó formulando una solución de este tipo, según la cual, aunque el universo en su totalidad está en equilibrio térmico, que es el estado más probable, y no hay, por tanto, ninguna base física para el sentido temporal antes-después, no es del todo imposible que algunas regiones del universo se aparten del equilibrio general durante un período relativamente breve de tiempo y evolucionen hasta recuperarlo. Según la sugerencia de Boltzmann, vivimos en una de esas regiones y a ello se deben nuestras convicciones acerca de asimetría del tiempo (L. Boltzmann 1964, p. 446). En los dos apartados siguientes discutiré dos posibles soluciones a estos problemas suscitados por la reducción de la termodinámica a mecánica estadística.

\section{INTERFERENCIAS}

Existe una explicación de la irreversibilidad termodinámica que se basa en el contraste existente entre la simplicidad de los modelos idealizados que las

5 Véanse L. Sklar 1993, p. 417 y 1995a; H. Price 1996, capítulo. 2; y C. Callender 1998.

6 Debo prescindir en este trabajo de la discusión relativa a si la irreversibilidad termodinámica que conocemos exige la hipótesis de un pasado de baja entropía que se remontaría a los orígenes del universo. Véanse al respecto H. Price 1996, capítulo 4; y C. Callender 1998 y 2004. 
teorías contemplan y la complejidad de los fenómenos reales a que se aplican. Más concretamente, se basa en el hecho de que, mientras que los referentes ideales de las teorías físicas fundamentales, como la mecánica clásica, son sistemas aislados, supuestamente libres de perturbaciones e interferencias, los sistemas reales están sujetos a la influencia de su entorno, con el que inevitablemente interactúan. Imaginemos que tenemos ante nosotros una masa suspendida en el extremo de un resorte y que la bajamos, estirando el resorte, hasta la posición $p$ y luego la soltamos. Si se tratara de un soporte teóricamente perfecto, sin rozamiento, y se pudiera anular la resistencia que oponen las moléculas del aire circundante, la física fundamental nos dice que la masa empezaría a oscilar indefinidamente, bajando una y otra vez hasta la posición $p$ en que la soltamos. Sin embargo, una masa en un resorte real muestra el comportamiento irreversible que todos conocemos, porque las imperfecciones del resorte y la resistencia del aire producen una continua disminución de la velocidad de sus oscilaciones. Algo similar ocurriría -defiende la mencionada explicacióncon el comportamiento, por ejemplo, de un gas que, después de haber estado confinado en una esquina de un recipiente, llega a ocupar ya gran parte de su volumen. Según los principios de la mecánica, no es del todo imposible que el gas interrumpa este proceso de expansión y vuelva a concentrarse espontáneamente en esa misma esquina, disminuyendo así su entropía. Pero al admitir esta posibilidad no se tiene en cuenta la inevitable acción que sobre el gas ejercen las moléculas de las paredes del recipiente y, a través de ellas, el entorno de éste. Estas influencias serían las responsables de que el gas exhiba su típico comportamiento termodinámico y llegue a ocupar todo el recipiente. Por esto dice Denbigh que «el concepto de reversibilidad sigue siendo una idealización útil para fines teóricos», pero los sistemas reales se comportan de modo irreversible (K. G. Denbigh 1989b, p. 507).

El problema es que, si las leyes fundamentales de la mecánica son universales, como se supone, se deben aplicar también a las interacciones de un sistema con su medio, y dado que estas leyes son invariantes respecto a la inversión temporal (en adelante, IIT), no es imposible que el proceso que experimenta el sistema junto con su medio sea también reversible. Es decir, que si contemplamos ahora no sólo las moléculas del gas sino también las del recipiente y su entorno inmediato, sigue siendo nomológicamente posible que, con la consiguiente inversión de los movimientos de todas ellas, el gas acabe concentrándose en una esquina del recipiente. Por tanto, mientras las leyes sean las mismas, la mera ampliación de los límites del sistema en consideración no implica un cambio sustancial en la situación ni facilita una solución del problema. Ciertamente, ahora la inversión del proceso resulta mucho más complicada aún, porque afecta a un número mucho mayor de elementos, pero este hecho sólo viene a reforzar la convicción, a que ya llegó Boltzmann, de que tal inversión es tremendamente improbable. 
Por otra parte, para que las interferencias provenientes del entorno resulten relevantes en la explicación de la tendencia general al crecimiento de entropía es preciso suponer que si no existiesen tales influencias, los fenómenos observados serían diferentes, esto es, la entropía no crecería del modo que conocemos por experiencia. Sin embargo no diríamos que este condicional resulta aceptable ¿Acaso si, en el ejemplo anterior, se anulase la acción del entorno, el gas no se extendería por todo el recipiente? Es más, tengamos en cuenta que no sólo las leyes fundamentales de la física contemplan situaciones ideales. La segunda ley de la termodinámica, siendo una ley fenomenológica, sólo se aplica propiamente a entidades tan poco reales como los sistemas perfectamente aislados, de modo que son precisamente las interferencias del medio las que explican, en última instancia, los comportamientos de sistemas en los que la ley no se cumple. En suma, no parece que la alusión a las perturbaciones procedentes del entorno ofrezca una explicación satisfactoria de la irreversibilidad que observamos en los procesos termodinámicos.

\section{DisOLUCIÓN DEL PROBLEMA}

El problema de la justificación de la segunda ley de la termodinámica parte, como hemos visto, del supuesto de que la mecánica clásica es IIT, y otro intento de solución consiste precisamente en negar tal supuesto. Es lo que hace Hutchison en su (1993). Admite que, ciertamente, la mecánica clásica contiene leyes IIT. Se trata de sus leyes consideradas fundamentales o principios, como la ley de gravitación o la segunda ley de Newton, que sólo contemplan fuerzas simétricas, esto es, fuerzas conservativas que dependen únicamente de la distancia entre los objetos y dan lugar a fenómenos reversibles. Pero contiene también leyes no-IIT, que se aplican a fuerzas disipativas, que son las más comunes en el nivel macroscópico: fuerzas, como la de fricción, que dependen de las velocidades de los elementos que interactúan y que producen procesos irreversibles. Y subraya Hutchison que tales procesos son propiamente mecánicos, que sus simulaciones son «paradigmáticas del corpus mecánico-clásico» (K. Hutchison 1993, p. 310) y que los cálculos acerca de ellos son del tipo de cálculos que todo estudiante de mecánica debe dominar y que los ingenieros aplican rutinariamente. Por tanto, la mecánica clásica acoge tanto procesos reversibles como irreversibles. Así, en el ejemplo anterior del movimiento de una masa suspendida en un resorte ideal y sin interferencias será la segunda ley de Newton la que dé cuenta de este fenómeno reversible, pero si complicamos el modelo incluyendo la fricción con las moléculas del entorno de modo que el proceso resultante sea irreversible, la ley que lo describe será una ley asimétrica, como es el caso de la ecuación de movimiento de un oscilador amortiguado. Con esta premisas concluye Hut- 
chison que la mecánica clásica es una teoría neutra respecto a la reversibilidad de los procesos de que se ocupa, un simple algoritmo que se aplica por igual a fuerzas conservativas, que producen comportamientos reversibles, y a fuerzas disipativas, que dan lugar a fenómenos irreversibles.

Según lo anterior, cabría pensar que el argumento de Hutchison consiste en negar que la mecánica clásica sea una teoría IIT basándose en la existencia de multitud de procesos genuinamente mecánicos, como el movimiento de la masa en nuestro resorte amortiguado, cuya inversión no se ha observado nunca. Si así fuera, su razonamiento carecería de relevancia, porque cuando se afirma que la mecánica clásica es IIT no se quiere decir que todos los fenómenos mecánicos han de invertirse, sino que su inversión es dinámicamente posible y, por tanto, tratándose de una mera posibilidad, los hechos que puedan esgrimirse en su contra no llegan a constituir verdaderos contraejemplos. ${ }^{7}$ Sin embargo, lo que Hutchison defiende es que los fenómenos mecánicos a que se refiere no son sólo procesos cuya inversión nunca hemos observado, sino procesos nomológicamente irreversibles debido al tipo de fuerzas que los provocan: fuerzas asimétricas, tan importantes, al menos, como las que rigen en los procesos reversibles.

La extendida creencia en que la mecánica clásica es IIT va unida, según Hutchison, a una actitud «fundamentalista»que centra la atención en la física fundamental, en cuyo dominio se suele considerar que las fuerzas generan movimientos reversibles, y concede muy poca importancia a los fenómenos mecánicos irreversibles que se producen en el nivel macroscópico, entendiendo que las fuerzas que los provocan son reducibles en última instancia a las fuerzas simétricas fundamentales que actúan en la microestructura. Esta actitud fundamentalista obliga, por tanto, a considerar teóricamente reversibles procesos como la conducción del calor, la expansión de los gases o las mezclas de líquidos, es decir, todos los fenómenos termodinámicos, y no puede eludir las objeciones a que tuvo que hacer frente Boltzmann. Pero, según Hutchison, la tesis de que la microestructura del mundo es fundamentalmente reversible no pasa de ser una opinión acerca de la naturaleza, un supuesto ontológico añadido artificialmente a la teoría, de modo que cuando, a partir de él, se dividen las fuerzas y leyes de una teoría en fundamentales y fenomenológicas, se corre el riesgo de estar haciendo una división puramente arbitraria. Y no es que podamos decir que esta tesis sea falsa, continúa Hutchison, sino que, al referirse a «la naturaleza de las fuerzas que actúan en este universo, es extraña a la mecánica clásica; una teoría que, sin necesidad de modificación alguna, debería ser igualmente aplicable en un universo diferente, en el que los procesos fundamentales fueran irreversibles. Por tanto, para Hutchison, la afirmación de que la mecánica clásica

7 En esto consiste sustancialmente la crítica de Savitt a Hutchison (S. F. Savitt 1994). 
es IIT es resultado de «una confusión entre las características de una teoría y las características del mundo de que trata la teoría» (K. Hutchison 1993, pp. 318 y 315). Es obvio que, desde esta concepción de las teorías científicas, no tiene sentido el problema con que se encontró Boltzmann y que ha preocupado y preocupa a muchos físicos y filósofos. Desde tal punto de vista, este problema queda disuelto como un pseudoproblema producido por la tendencia a desnaturalizar el contenido de la mecánica clásica con consideraciones ontológicas que le son ajenas.

No es difícil estar de acuerdo con Hutchison en que, como cuestión de hecho, hay procesos mecánicos que se invierten y otros que no lo hacen nunca y hay leyes mecánicas IIT y no-IIT. Y no le falta razón al subrayar que la afirmación de que la mecánica clásica es una teoría IIT responde a cierto supuesto ontológico, dado que tal afirmación incluye la creencia en que determinadas leyes de la teoría constituyen el núcleo de su contenido explicativo. Sin embargo, sus ideas acerca de la neutralidad ontológica de la mecánica clásica resultan menos convincentes. Su valoración de las teorías físicas como meros instrumentos de cálculo, carentes de implicaciones ontológicas responde, como se sabe, a una vieja concepción filosófica discutible y largamente discutida desde los orígenes de la ciencia moderna. No puedo entrar aquí detenidamente en esa polémica, pero defenderé brevemente que la tesis de que las interacciones fundamentales de la naturaleza son reversibles y de que, en consecuencia, la mecánica clásica es IIT no es, como pretende Hutchison, una tesis arbitraria, injustificada y ajena a esta teoría.

Tomar como básicas las interacciones entre partículas que dependen de las posiciones relativas de éstas y admitir que, por tanto, la microestructura del mundo es fundamentalmente reversible puede considerarse resultado de convicciones tan poco extrañas en la física de los últimos siglos como a) la hipótesis atómica, que forma parte del paradigma actual de la física; b) la validez universal del principio de conservación de la energía; y c) la simplicidad que debemos exigir en las hipótesis de carácter ontológico. La segunda convicción es directamente responsable de la creencia en que las llamadas fuerzas disipativas no existen realmente en cuanto tales y que sus pretendidos efectos son explicables mediante fuerzas conservativas que actúan entre elementos más básicos. Si el concepto de disipación invita a pensar en la «pérdida» de parte de la energía de un sistema como resultado, por ejemplo, de la fricción con el medio, la convicción en la validez global del principio de conservación nos obliga a considerar ese fenómeno como uno en que la disminución de energía del sistema tiene su exacta contrapartida en el incremento de energía en las partículas del medio. Las llamadas fuerzas disipativas no son, por tanto, genuinas fuerzas naturales porque sus efectos se pueden explicar perfectamente en términos de la acción de fuerzas conservativas. Como señala Callender, el motivo que nos lleva considerar 
a las fuerzas disipativas como meramente aparentes es en definitiva el mismo que, una vez que conocemos el significado del concepto de inercia y admitimos que el espacio es homogéneo, nos obliga a negar que existan realmente fuerzas centrífugas o de Coriolis (C. Callender 1995). Por otra parte, no cabe duda de que esta concepción de la mecánica tiene el valor metodológico de ser más simple, dado que postula menos tipos de fuerzas en la naturaleza y es capaz de explicar unificadamente un gran número de fenómenos aparentemente diferentes. Es cierto que la simplicidad de las hipótesis no es señal de su verdad, pero cuando se trata de hipótesis ontológicas, la parsimonia explicativa constituye una característica especialmente valiosa. En este sentido cabe decir que el punto de vista de Hutchison es el de una ontología menos arriesgada (admite mayor variedad de entidades y relaciones) pero mucho más compleja.

Vistas la ideas de Hutchison acerca de la naturaleza de las teorías científicas, se puede decir que su intento de disolver el problema de Boltzamnn podría ser más radical. Para tal disolución no es necesario que se moleste en refutar la hipótesis de que la mecánica clásica es IIT, sino que le basta con negar el supuesto que generó el problema, es decir, el supuesto de que el calor es energía cinética y, por tanto, que los fenómenos y leyes de la termodinámica son analizables en términos mecánicos. Si, como Hutchison sostiene, las teorías físicas no deben incorporar ninguna hipótesis ontológica acerca de la naturaleza de la materia ni de las fuerzas que se dan en ella, la hipótesis relativa a la naturaleza mecánica del calor no formaría parte de la física, y los problemas conceptuales que se derivan de ella no serían genuinos problemas científicos. De hecho, científicos instrumentalistas del finales del siglo XIX como Mach y Duhem no admitían la reducción de la termodinámica a mecánica estadística.

Aquí sólo he comentado o discutido algunos intentos de solución al problema de la explicación de la irreversibilidad de los procesos termodinámicos, pero creo que las dificultades con que, como hemos visto, tales intentos se encuentran dan una idea del alcance del problema y explican en buena medida que éste siga siendo aún una cuestión pendiente en la física teórica y su filosofía.

\section{IV. ¿QUÉ NOS QUEDA DE LA ASIMETRÍA DEL TIEMPO?}

Los fracasos en la explicación de la ley de entropía condujeron a Boltzmann, como sabemos, a la conclusión de que el tiempo es realmente simétrico, como el espacio, y a la hipótesis un tanto ad hoc de que la asimetría temporal que observamos a nuestro alrededor es excepcional y efímera. Sin embargo, para llegar a tal conclusión a partir de las múltiples dificultades o, si se quiere, de la imposibilidad de explicar la irreversibilidad termodinámica, es necesaria otra premisa: el supuesto de que todos los procesos naturales irreversibles que podrían 
explicar la asimetría del tiempo son básicamente procesos termodinámicos, o sea, que los diferentes procesos irreversibles físicos, biológicos o mentales son plenamente definibles en términos de incremento de entropía. No cabe duda de que muchos de estos procesos, incluidos los biológicos y los mentales, involucran fenómenos físico-químicos que son inequívocamente termodinámicos, pero lo que esta premisa supone es que todo proceso irreversible es en sí mismo termodinámico, es decir, que existe una identificación entre orden temporal y orden entrópico del mismo tipo que la que existe entre ondas luminosas y ondas electromagnéticas o entre calor y energía cinética media. De hecho, Boltzmann defendía esta reducción entrópica de la irreversibilidad. Afirmaba que, del mismo modo que, en el espacio que rodea a nuestro planeta, el sentido de «hacia abajo» se identifica necesariamente con aquél en que actúa la atracción gravitatoria sobre los cuerpos, la orientación temporal hacia el futuro viene indicada por el incremento de entropía (L. Boltzmann 1964, pp. 446-7). Así, al igual que en una región del espacio donde no exista una fuerza gravitatoria neta no habría un sentido de «hacia abajo», allí donde se haya alcanzado el equilibrio termodinámico no habría un sentido temporal antes-después. Este punto de vista reduccionista ha encontrado notable eco en muchos filósofos de la ciencia, entre ellos, Reichenbach, Grünbaum, Costa de Beauregard y Smart, pero, al menos hasta ahora, no pasa de ser una atractiva hipótesis unificadora sin suficiente confirmación o un programa no sólo incompleto, sino susceptible de crítica incluso en sus aparentes logros parciales. ${ }^{8}$ Téngase en cuenta, por ejemplo, que un requisito elemental en la aplicación del concepto de entropía a fenómenos no termodinámicos consiste en respetar su significado propio, ya sea en su primera versión, es decir, como medida de la dificultad de obtener energía de un sistema o, en la versión de Boltzmann, esto es, como el paso de un sistema al macroestado más probable; sin embargo es muy discutible que se cumpla este requisito cuando, como es tan frecuente en las aplicaciones de la noción de entropía a procesos no termodinámicos, se define ésta en términos de desorden, desorganización o falta de información (K. G. Denbihg 1989a; P. Horwich 1987, p. 83). En resumen, mientras el proyecto de reducción entrópica de las distintas flechas del tiempo no obtenga resultados más convincentes de los logrados hasta ahora, las dificultades relativas a la explicación teórica de la irreversibilidad termodinámica no constituyen un serio obstáculo para la explicación de la asimetría del tiempo en términos de procesos irreversibles. 


\section{LA ASIMETRÍA DEL TIEMPO EN NOSOTROS}

Contemplemos, finalmente, la posibilidad de que nuestra convicción acerca de la asimetría del tiempo dependa en última instancia no de lo que ocurre en la naturaleza, sino de nosotros mismos, de nuestro modo de percibir y concebir los fenómenos naturales. En lo que sigue consideraré esta hipótesis tal como aparece en Price 1996. En los primeros capítulos de este libro, Price subraya los insalvables obstáculos con que se encontró Boltzmann al pretender explicar la flecha del tiempo termodinámica, argumenta además contra las supuestas asimetrías temporales de los fenómenos de radiación y de la expansión del universo y acaba defendiendo que no existe propiamente una flecha del tiempo en el mundo físico. Luego, en los capítulos 6 y 7, se plantea si la asimetría temporal de la causalidad puede constituir realmente un fundamento para la asimetría del tiempo, es decir, si la orientación antes-después tiene su razón de ser en el hecho general de que las causas preceden a sus efectos. Pero, en el tratamiento de este asunto Price defiende un concepto de causalidad, según el cual, la relación entre causa y efecto no es sino la relación entre medios y fines que nosotros, en cuanto seres capaces de acciones intencionales y libres, proyectamos en la naturaleza. Por tanto, decir que A es causa de B significa simplemente que A es un medio eficaz con el que podríamos conseguir que ocurriera B $(\mathrm{H}$. Price 1996, p. 157). Se trata de una versión antropocéntrica de la causalidad, según la cual, el origen de este concepto reside en nuestra experiencia de conseguir unas cosas haciendo otras, y la razón de la asimetría causal no está en el mundo sino en nosotros, que como agentes somos temporalmente asimétricos, o sea, podemos influir sobre sucesos futuros pero no modificar los sucesos pasados. ${ }^{9}$ En apoyo de esta idea, compara Price la causalidad con las cualidades secundarias, como el color o el sabor, afirmando que al igual que éstas resultan de las características de nuestro peculiar aparato sensorial, la relación causal y su asimetría temporal son productos de nuestra perspectiva como agentes, de nuestra condición de seres capaces de intervenir y modificar voluntariamente nuestro medio. Por tanto, igual que en un mundo en el que no hubiera seres con un aparato sensorial como el nuestro no habría colores ni sabores, en un mundo sin seres capaces de acciones intencionales no habría ni causas ni efectos. Esta concepción de la causalidad y de su asimetría temporal sirve de base a la tesis de Price de que nuestra convicción acerca de la orientación del tiempo, de la asimetría entre el pasado y el futuro, lejos de encontrar un soporte objetivo en

9 Esta noción de causalidad es una variante de la llamada teoría de la manipulabilidad, defendida, entre otros, por Gasking y von Wright, y de los análisis de la relación causal en términos de intervención humana, como los de Glymour, Pearl o Woodward. No obstante Price se considera mucho menos «objetivista» que estos autores (H. Price 2007, p. 268). 
las relaciones causales, tiene su explicación en nosotros mismos, es subjetiva y da origen los prejuicios que nos impiden comprender el tiempo simétrico de la física (H. Price 1996, pp. 8-9).

La definición de la causalidad en términos de manipulación humana ha sido objeto de diversas críticas. Se objeta, por ejemplo, que es circular, porque la misma acción humana supone ya la idea de causalidad, o que no da razón de nuestras afirmaciones acerca de relaciones causales en dominios donde la acción humana es imposible. Pero, prescindiré aquí de estas objeciones y me limitaré a mostrar brevemente hasta dónde puede llegar Price en su pretensión acerca del carácter meramente antropocéntrico o subjetivo de la noción de causalidad, hasta qué punto puede mantener que «la asimetría causal refleja una asimetría en nosotros, no una asimetría en el mundo externo» (H. Price 1996, p.161). En primer lugar, estaremos de acuerdo en que, si la relación causal con su asimetría propia depende, como las cualidades secundarias, de algún rasgo de la naturaleza humana, en concreto, de nuestra condición de agentes, entonces, al igual que animales con un aparato sensorial distinto del nuestro perciben el mundo modo distinto, otros seres intencionales con capacidades de acción diferentes a las nuestras tendrían una idea diferente de causalidad. Ante esta cuestión, Price, aunque sostiene, como sabemos, que en un mundo en el que sus habitantes inteligentes carecieran por completo de la capacidad de modificar su entorno «nada procedería de nada», porque tales criaturas no podrían desarrollar ninguna noción de causalidad, no llega a admitir que una persona con capacidades de manipulación de la naturaleza diferentes a las habituales tuviera un concepto de causalidad diferente al nuestro. La causalidad es, para Price, mucho menos sensible que las cualidades secundarias a las diferencias en la condición humana: se trataría de una cualidad secundaria bastante sui generis, porque «es significativamente más objetiva que el color o el sabor» (P. Menzies and H. Price 1993, pp. 199-201). Pero veamos qué margen de objetividad está dispuesto a conceder Price a la causalidad y a su orientación temporal. $\mathrm{Si}$, como pretende, el concepto de causalidad surge de nuestra perspectiva de agentes intencionales, ¿por qué es inútil que intentemos actuar sobre el pasado para modificarlo?, ¿por qué los agentes intencionales somos seres temporalmente asimétricos? A estas cuestiones responde que los humanos, en cuanto seres macroscópicos, estamos sujetos a la asimetría termodinámica y que en asuntos prácticos, como son nuestras acciones intencionales, la ley de entropía nos limita como si fuera una genuina ley de la física. Pero matiza Price que esta imposibilidad nuestra para cambiar el pasado, al depender de nuestra condición de agentes macroscópicos, constituiría, aunque no seamos conscientes de ello, una orientación temporal meramente fenomenológica y contingente, como la misma flecha del tiempo termodinámica, de modo que tal imposibilidad no sería «una verdad absoluta acerca del mundo» sino una verdad desde nuestra perspectiva de agentes en el mundo (Price 1996, pp. 160, 167). 
Como podemos ver, Price parece conceder una buena dosis de objetividad a la orientación temporal de la relación causal y, por consiguiente, a nuestra incapacidad para modificar el pasado. En efecto, podemos quemar un trozo de madera, pero la irreversibilidad termodinámica no nos permite recuperarlo a partir de sus cenizas; podemos fundir un trozo de hielo introduciéndolo en agua, pero no deshacer el mismo proceso hasta recuperar ese trozo de hielo. La orientación temporal de la causa y el efecto no es asunto de elección, sino que nos viene impuesta por la naturaleza. La insistencia de Price en que, a pesar de ello, tal orientación sigue siendo una cuestión de perspectiva, es decir, de nuestra perspectiva como agentes, se apoya en dos premisa. La primera es el carácter fenomenológico y contingente de la termodinámica. Pero esta premisa no contribuye en nada a la conclusión de Price. El hecho de que la ley de entropía sea una ley derivada de los principios de la mecánica y no se conozcan con certeza sus condiciones iniciales no dice nada contra el carácter objetivo de la orientación temporal que la irreversibilidad termodinámica impone a nuestras acciones, ni mucho menos convierte a esta orientación en un producto de nuestra capacidad para modificar nuestro entorno. La otra premisa, que Price no menciona pero resulta imprescindible para su conclusión, es la tesis reduccionista de que todos los procesos naturales irreversibles son en última instancia termodinámicos, es decir, que todas las flechas del tiempo son interpretables en términos de incremento de entropía. Pero aun en el caso de que esto fuera cierto en el mundo físico, como Price cree haber demostrado, existen multitud de procesos irreversibles en el nivel biológico o en el mental que indican una orientación privilegiada del tiempo, que sitúan los resultados de nuestras acciones en el futuro y que, como vimos anteriormente, no tienen por qué ser considerados fenómenos termodinámicos. Podemos contribuir al desarrollo de una planta abonando la tierra, regándola, etc., pero no hay modo de conseguir que vuelva a ser una semilla. Y nada nos obliga a admitir que el crecimiento de una planta sea un proceso termodinámico.

En suma, independientemente de que la irreversibilidad termodinámica sea fenomenológica y contingente y de nuestra condición de seres macroscópicos, la orientación temporal de nuestras acciones y la prioridad temporal de las causas sobre los efectos tienen una base en la naturaleza. No le falta razón a Price al sostener que nuestra convicción acerca de la asimetría del tiempo está relacionada con nuestra capacidad de intervenir eficazmente en nuestro entorno, pero no porque tal asimetría sea antropocéntrica o subjetiva, como él pretende, sino porque es en nuestras intervenciones, desde las más sencillas hasta las más complejas, donde comprobamos la existencia de múltiples procesos naturales irreversibles que son independientes de nuestras intenciones como agentes. Con esto no pretendo haber mostrado que es imposible una explicación de la asimetría del tiempo en términos subjetivos, sino tan sólo que una explicación 
basada en nuestra condición de agentes intencionales como la que Price propone no es una buena candidata para ese propósito.

\section{CONCLUSIÓN}

El problema de la explicación teórica de la irreversibilidad termodinámica sigue aún pendiente de solución, pero, a menos que se admita como suficientemente probada la discutible hipótesis de que todo proceso irreversible es, en última instancia, un fenómeno de incremento de entropía, dicho problema no afecta sustancialmente al punto de vista que defiende la explicación de la asimetría del tiempo en términos de procesos irreversibles. Este punto de vista se ha visto reforzado en este artículo con el análisis del intento de Price de ofrecer una versión meramente antropocéntrica de la asimetría del tiempo, puesto que si eliminamos en su argumentación el supuesto tácito de que todo proceso irreversible es fundamentalmente termodinámico, la conclusión es sencillamente que la orientación temporal de nuestras acciones, esto es, nuestra incapacidad para cambiar el pasado, reside, en definitiva, en la irreversibilidad que exhiben multitud de procesos naturales.

\section{REFERENCIAS BIBLIOGRÁFICAS}

BERRY, S. 1995: «Entropy, Irreversibility and Evolution», Journal of Theoretical Biology, 175, pp. 197-202.

BOLTZMANN, L. 1877: «On the Relation of a General Mechanical Theorem to the Second Law of Thermodynamics» en S. Brush (ed.), Kinetic Theory. Volume 2: Irreversible Processes. Oxford: Pergamon Press, 1966, pp. 188-193.

BOLTZMANN, L. 1964: Lectures on Gas Theory. Berkeley: University of California Press. English translation of L. Boltzmann, Vorlesungen über Gastheorie. Leipzig: J. A. Barth, 1896-1898.

CALLENDER, C. 1995: «Discussion: The Metaphysics of Time Reversal: Hutchison on Classical Mechanics», British Journal for Philosophy of Science, 46, pp. 331340.

CALLENDER, C. 1998: «The View from No-when», British Journal for Philosophy of Science, 49, pp. 135-159.

CALLENDER, C. 2004: «Measures, Explanation and the Past: Should 'Special' Initial Conditions be Explained?», British Journal for Philosophy of Science, 55, pp. 195-217.

DENBIGH, K. G. 1989a: «Note on Entropy, Disorder and Disorganization», British Journal for Philosophy of Science, 40, pp. 323-332.

DENBIGH, K. G. 1989b: «The Many Faces of Irreversibility», British Journal for Philosophy of Science, 40, pp. 501-518. 
DIEKS, D. (ed.) 2006: The Ontology of Spacetime. Amsterdam: Elsevier.

HORWICH, P. 1987: Asymmetries in Time. Cambridge (Mass.): Massachusetts Institute of Technology.

HUTCHISON, K. 1993: «Is Classical Mechanics Really Time-reversible and Deterministic?», British Journal for Philosophy of Science, 44, pp. 307-323.

LE POIDEVIN, R. 1998: «The Past, Present and Future of the Debate about Tense», en R. Le Poidevin, (ed.): Question of Time and Tense. Oxford: Oxford University Press, pp. 13-42.

MELLOR, D. H 1998: Real Time II. London and New York: Routledge.

MENZIES, P. and PRICE, H. 1993: «Causation as a Secondary Quality», British Journal for Philosophy of Science, 44, pp. 187-203.

PRICE, H. 1996: The Time's Arrow and Archimedes's Point. Oxford: Oxford University Press.

PRICE, H. 2007: «Causal Perspectivalism», en H. Price and R. Corry (eds.), Causation, physics, and the constitution of reality. Oxford : Clarendon Press, pp. 250-292.

SAVITT, S. F. 1994: «Is Classical Mechanics Time Reversal Invariant?», British Journal for Philosophy of Science, 45, pp. 907-913.

SAVITT, S. F. (ed.) 1995: Time's Arrow Today, Cambridge University Press, Cambridge.

SKLAR, L. 1993: Physics and Chance. Cambridge: Cambridge University Press.

SKLAR, L. 1995a: «The elusive object of desire: in pursuit of the kinetic equations and the Second Law», en Savitt (ed.), 1995, pp. 192-216.

SKLAR, L.1995b: «Time in experience and in theoretical description of the world», en Savitt (ed.), 1995, pp. 217- 229.

Sebastián Álvarez Toledo es profesor titular de Lógica y Filosofía de la Ciencia en la Facultad de Filosofía de la Universidad de Salamanca.

Publicaciones recientes:

«Interferencias y asimetría causal», Actas del IV Congreso de la Sociedad de Lógica, Metodología y Filosofía de la Ciencia en España. Valladolid, 2004, 121-123.

«Racionalidad y diversidad en la ciencia», en A. R. Pérez Ransanz y A. Velasco (eds.) Racionalidad teórica y racionalidad práctica, UNAM, México, 2007.

Dirección postal:

Facultad de Filosofía. Campus M. de Unamuno. Universidad de Salamanca. 37007 Salamanca.

Dirección electrónica: sat@usal.es 